\title{
Linear General Position (i.e. Arcs) for Zero-Dimensional Schemes Over a Finite Field
}

\author{
Edoardo Ballico \\ Department of Mathematics, University of Trento, 38123 Povo (TN), Italy \\ E-mail: ballico@science.unitn.it
}

Received: 8 April 2021; Revised: 22 July 2021; Accepted: 2 August 2021

\begin{abstract}
We extend some of the usual notions of projective geometry over a finite field (arcs and caps) to the case of zero-dimensional schemes defined over a finite field $\mathbb{F}_{q}$. In particular we prove that for our type of zero-dimensional arcs the maximum degree in any $r$-dimensional projective space is $r(q+1)$ and (if either $r=2$ or $q$ is odd) all the maximal cases are projectively equivalent and come from a rational normal curve.
\end{abstract}

Keywords: arcs, zero-dimensional scheme, linear general position sets

MSC: 14N05, 12E99, 12F99

\section{Introduction}

There is a huge literature on arcs in projective spaces over a finite field, in large part started by Segre B, his collaborators, his students and their descendants ([1-13]). In this paper, we consider zero-dimensional schemes instead of finite sets and consider for them the notions of arcs and caps.

Fix a prime field $F$, i.e. either $F=\mathbb{Q}$ or $F=\mathbb{F}_{p}$ for some prime $p$, and let $\bar{F}$ be the algebraic closure of $F$. Take any field $K$ such that $F \subseteq K \subseteq F$. Of course, we are mainly interested in the case $F=\mathbb{F}_{p}$ and $K:=\mathbb{F}_{q}$, but we state in a general set-up the statements that do not use the finiteness of the field $K$.

Let $S \subset \mathbb{P}^{n}(K)$ be a finite set. We say that $S$ is in linear general position (in [14, p. 109] and [15, Lemma 1.1] it is called General Position) or that it is an arc (terminology of [5-11]) if all $A \subseteq S$ such that \#A $\leq n+1$ are linearly independent.

Definition 1.1 Let $Z \subset \mathbb{P}^{r}(\bar{F})$ be a zero-dimensional scheme defined over $K$. We say that $Z$ is in linear general position with respect to $K$ or that it is an arc with respect to $K$ if for each $t \in\{1, \ldots, r-1\}$ and each $t$-dimensional linear subspace $V \subset \mathbb{P}^{r}(\bar{F})$ defined over $K$ we have $\operatorname{deg}(V \cap Z) \leq t+1$.

Definition 1.2 Let $Z \subset \mathbb{P}^{r}(\bar{F})$ be a zero-dimensional scheme defined over $K$. We say that $Z$ is rnc with respect to $K$ or just rnc if each connected component $W$ of $Z$ satisfies $\operatorname{deg}(W) \leq r+1$ and there are $o \in \mathbb{P}^{r}(K)$ and a rational normal curve $C \subset \mathbb{P}^{r}(\bar{F})$ defined over $K$ such that $o \in C(K)$ and $W$ is the effective $\operatorname{divisor} \operatorname{deg}(W) o$ of $C(\bar{F})$. We will say that $Z$ is $\leq t$-rnc if it is rnc and each connected component of $Z$ has degree $\leq t$. We say that $Z$ is an + -rnc if it is an $\leq 2$-rnc.

The acronym "rnc" stands for "rational normal curve". In Definition 1.2 we assumed $\operatorname{deg}(W) \leq r+1$ for each

Copyright (C2021 Edoardo Ballico.

DOI: https://doi.org/10.37256/cm.232021864

This is an open-access article distributed under a CC BY license

(Creative Commons Attribution 4.0 International License)

https://creativecommons.org/licenses/by/4.0/ 
connected component $W$ of $Z$, because we use Definition 1.2 in the definition of arcs and to be $Z$ (and hence $W$ ) an arc, this is a necessary condition. Often we will omit "with respect to $K$ " because a connected scheme defined over $K$ is an rnc with respect to $K$ if and only if it is rnc with respect to some field $K^{\prime} \supset K$.

Definition 1.3 Let $V \subset \mathbb{P}^{n}(\bar{F})$ be an $x$-dimensional vector space defined over $K$. An $x$-dimensional osculating data defined over $K$ and spanning $V$ is a pair $(C, o)$, where $C$ is a rational normal curve of $V$ defined over $K$ and $o \in C(K)$.

Let $Z \subset \mathbb{P}^{r}(\bar{F})$ be a zero-dimensional scheme. We say that $Z$ is curvilinear if each connected component of $Z$ is either a point or it has 1-dimensional Zariski tangent space.

Definition 1.4 Let $V \subset \mathbb{P}^{n}(\bar{F})$ be an $x$-dimensional vector space defined over $K$. An $x$-dimensional osculating data defined over $K$ spanning $V$ is a degree $x$ connected curvilinear scheme $Z$ such that $V$ is the minimal linear space containing $Z$ and for each $z \in\{1, \ldots, x+1\}$ the only degree $z$ subscheme of $Z$ is defined over $K$.

Definition 1.5 Fix an integer $v \in\{0, \ldots, r\}$, a $v$-dimensional linear subspace $V \subseteq \mathbb{P}^{r}(\bar{F})$ defined over $K$ and $o \in$ $V(K)$. The double point $(2 o, V)$ of $o$ in $V$ is the closed subscheme of $V$ with $\left(\mathcal{I}_{o}, V\right)^{2}$ as its ideal sheaf. A zero-dimensional scheme $Z \subset \mathbb{P}^{r}(\bar{F})$ defined over $K$ is called a scheme of double points or a scheme of double points over $K$ if all its connected components are defined over $K$ and all its connected components are of the form $(2 o, V)$ for some $v, V$ and $o$ (the integers $v$ 's may be different for different connected components). A zero-dimensional scheme of $\mathbb{P}^{r}(\bar{F})$ is said to be of mixed type if each of its connected components is defined over $K$ and it is either rnc or a double point.

Definitions 1.4 and 1.5 give the same data if $K$ is perfect (see Lemma 2.11).

Remark 1.6 Take $v, V$, and $o$ as in Definition 1.5 and set $Z:=(2 o, V) . Z$ is a zero-dimensional scheme defined over $K, \operatorname{deg}(Z)=v+1$ and $V$ is the linear span of $V$. Note that $v+1$ is the minimal degree of a zero-dimensional scheme spanning $V$. If $v \neq 0$, we may think of $Z$ as the object describing (from the point of view of linear dependence) all partial derivatives at $o$ with respect to the directions associated to the lines passing through $o$ and contained in $V$. Since $V$ is defined over $K$, there are $v$ lines through $o$ defined over $K$ and whose union spans $V$. Thus, if a mixed scheme $E$ is in linear general position and has a connected component $(2 o, V)$ with $\operatorname{dim} V=r$, then $E=(2 o, V)$ and hence $\operatorname{deg}(E)=r+$ 1 (Remark 2.10).

Remark 1.7 Every connected component $W$ of an rnc zero-dimensional scheme $Z$ is linearly independent. However, if $\operatorname{deg}(W)=r+1, Z$ is an arc if and only if $W=Z$. Thus to study arcs only +-rnc zero-dimensional schemes are interesting.

We prove the following results.

Theorem 1.8 Fix a prime integer $p$ and a $p$-power $q$.

(a) $2 q+2$ is the maximal degree of a + rnc arc of the plane defined over $\mathbb{F}_{q}$.

(b) Let $Z \subset \mathbb{P}^{2}\left(\mathbb{F}_{q}\right)$ be a + rnc arc over $\mathbb{F}_{q} . Z$ is complete if and and only if either $Z_{\text {red }}$ is complete or $q$ is even, $\operatorname{deg}(Z)$ $=q+1$ and $Z_{\text {red }}$ is an oval.

Theorem 1.9 Assume $q \geq r+3$. Let $z$ be the maximal degree of an $+\operatorname{rcn} \operatorname{arc} Z$ of $\mathbb{P}^{r}\left(\mathbb{F}_{q}\right), r \geq 2$. Then:

(1) $z=r(q+1)$.

(2) Assume $q$ odd. Then $\operatorname{deg}(Z)=r(q+1)$ if and only if each connected component of $Z$ has degree $r$ and $Z_{\text {red }}=$ $C\left(\mathbb{F}_{q}\right)$ for some rational normal curve $C$ defined over $\mathbb{F}_{q}$.

In the last section, we collect a few easy remarks concerning caps.

Our main motivation came from the use of zero-dimensional schemes to get results on the so-called $X$-rank, which has as a particular case the additive decomposition of a homogeneous polynomial and the tensor rank of a tensor ([2132]). In some of these papers, curvilinear schemes are the main actors. Small degree curvilinear schemes contained in low degree rational normal curves are key tools in [16-20, 25, 31]. The double points (Definition 1.6) are a fundamental tool for the computation of the dimensions of joins and secant varieties by the Terracini Lemma ([33, Corollary 1.11]).

As far as we know all results here are new, although certainly not deep, their proofs requiring just to combine classical results on finite geometry and tools used in the quoted papers on $X$-ranks.

\section{Preliminaries}

For any zero-dimensional scheme $Z$, let $Z_{\text {red }}$ denote its reduction, i.e. $Z_{\text {red }}$ is the maximal finite set contained in $Z$. 
The integer $\# Z_{\text {red }}$ is the number of connected components of $Z$.

Definition 2.1 An rnc arc $Z \subset \mathbb{P}^{r}(\bar{F})$ defined over $K$ is said to be a weakly complete arc or to be a weakly complete arc with respect to $K$ if there is no $b \in \mathbb{P}^{r}(K) Z_{\text {red }}$ such that $Z \cup\{b\}$ is an arc. $Z$ is called a complete arc (with respect to $K$ ) if it is not strictly contained in another rnc arc defined over $K$.

Remark 2.2 Let $Z \subset \mathbb{P}^{r}(\bar{F})$ be a zero-dimensional scheme. $Z$ is curvilinear if and only if each of its connected components is contained in an affine smooth curve. If $r \geq 3$ and $Z$ are curvilinear, then it is contained in a smooth curve $C \subset \mathbb{P}^{r}(\bar{F})$ defined over $\bar{F}$. Now assume that $Z$ is curvilinear, connected and defined over the perfect field $K$. Since $Z$ is curvilinear and connected, for each integer $z \in\{1, \ldots, \operatorname{deg}(Z)\}$ there is a unique degree $z$ subscheme $Z_{z}$ of $Z$. Since $Z$ is defined over $K$ and $Z_{z}$ is unique, Galois gives that $Z_{z}$ is defined over $K$.

Remark 2.3 Let $C \subset \mathbb{P}^{r}(\bar{F}), r \geq 2$, be a rational normal curve defined over $K$. Fix $o \in C(K)$ and any positive integer $x$. The divisor $Z_{x}:=x o$ of $C$ is a zero-dimensional scheme defined over $K$. Since $Z_{x} \subset C(\bar{F}), Z_{x}$ is in linear general position. Taking $x \gg 0$ and $K=\mathbb{F}_{q}$ we see why we assumed some restrictions for the rnc schemes.

Remark 2.4 If we consider non-curvilinear schemes, then further pathologies may occur. Fix $o \in \mathbb{P}^{r}(K)$ and let $Z$ denote the closed subscheme of $\mathbb{P}^{r}(\bar{F})$ with $\left(\mathcal{I}_{o}\right)^{2}$ as its ideal sheaf. $Z$ is a degree $r+1$ zero-dimensional scheme defined over $K$ and $\mathbb{P}^{r}(\bar{F})$ is the minimal linear space (over any field containing $K$ ) containing $Z$. Although $Z$ has very low degree, it is a weak blocking set over any field containing $K$, because for any $a \in \mathbb{P}^{r}(\bar{F}) \backslash\{o\}$ the line $\langle\{a, o\}\rangle$ (which is defined over any field containing $K$ and on which $a$ is defined) intersects $Z \cup\{a\}$ in a degree 3 scheme. Now we prove that $Z$ is not a blocking scheme. We prove the existence of a connected zero-dimensional scheme $W \supset Z$ defined over $K$ such that $\operatorname{deg}(W)=2^{r}$ and $\operatorname{deg}(L \cap W) \leq 2$ for every line $L \subset \mathbb{P}^{r}(\bar{F})$. Fix $r$ linearly independent hyperplanes $H_{1}, \ldots, H_{r}$ defined over $K$ such that $H_{1} \cap \cdots \cap H_{r}=\{o\}$. Let $Q_{i}$ be the rank 1 quadric hypersurface with the square of the equation of $H_{i}$ as its equation. Set $W:=Q_{1} \cap \cdots \cap Q_{r}$. Since each $H_{i}$ is defined over $K$, each $Q_{i}$ is defined over $K$ and hence $W$ is defined over $K$. Since $W_{\text {red }}=\{o\}, W$ is connected. Since $W$ is a complete intersection of quadric hypersurfaces, Bezout's theorem gives $\operatorname{deg}(W)=2^{r}$ and $\operatorname{deg}(L \cap W) \leq 2$ for every line $L \subset \mathbb{P}^{r}(\bar{F})$.

Remark 2.4 shows why we will not consider mixed schemes for arcs or caps. The next lemma shows that if a mixed scheme is an arc, then there are associated rnc schemes which are arcs with the same degree.

Lemma 2.5 Fix an integer $v \in\{1, \ldots, r\}$, a $v$-dimensional linear subspace $V \subseteq \mathbb{P}^{r}(\bar{F})$ defined and $o \in V(K)$. Set $W:=(2 o, V)$. Let $C \subset V$ be a rational normal curve of $V$ defined over $K$ and such that $o \in C(K)$. Let $E$ be the degree $v$ +1 zero-dimensional scheme with $\{o\}$ as its reduction. Let $Z \subset \mathbb{P}^{r}(\bar{F})$ be a zero-dimensional scheme whose connected components are defined over $K$ and such that $W$ is a connected component of $Z$. Set $Z^{\prime}:=(Z \backslash W) \cup E$. Then:

(i) $\operatorname{deg}(Z)=\operatorname{deg}\left(Z^{\prime}\right)$;

(ii) $Z^{\prime}$ is an $\operatorname{arc}$ if $Z$ is an arc.

Proof. Since $\operatorname{deg}(E)=\operatorname{deg}(W)$, then $\operatorname{deg}(Z)=\operatorname{deg}\left(Z^{\prime}\right)$. Assume that $Z$ is an arc and let $M \subset \mathbb{P}^{r}(\bar{F})$ a $t$-dimensional linear space defined over $K$. Set $Z^{\prime \prime}:=(Z \backslash W) \cap M, M^{\prime}:=M \cap V, W^{\prime}:=W \cap M$ and $E^{\prime}:=E \cap M$. Since $Z \cap M=Z^{\prime} \cap M=Z^{\prime \prime}$ if $o \notin M$, we may assume $o \in M$. Since $o \in M$, the definition of $W \operatorname{gives} \operatorname{deg}\left(W^{\prime}\right)=\operatorname{dim} M^{\prime}+1$, while the definition of $E$ as a connected rnc scheme gives $\operatorname{deg}\left(E^{\prime}\right) \leq \operatorname{dim} V^{\prime}+1$. Since $Z$ is an $\operatorname{arc}, \operatorname{deg}\left(Z^{\prime}\right)=\operatorname{deg}\left(Z^{\prime}\right)+\operatorname{deg}\left(E^{\prime}\right) \leq \operatorname{deg}\left(Z^{\prime}\right)+\operatorname{deg}(W)$ $\leq \operatorname{dim} M+1$. Thus $Z^{\prime}$ is an arc.

Consider the following elementary construction.

Proposition 2.6 Fix integers $r>s \geq[r / 2]>0$ and an $\leq s$-rnc arc $A \subset \mathbb{P}^{r}(\bar{F})$ over $K$ such that each connected components of $A$ has degree $s$ and $A$ is not weakly complete. Fix $o \in \mathbb{P}\left(\mathbb{F}_{q}\right) \backslash A_{\text {red }}$ such that $A \cup\{o\}$ is an arc. For any $a \in$ $A_{\text {red }}$, let $A_{a}$ be the connected component of $A$ with a as its reduction. Set $V_{a}:=\left\langle A_{a} \cup\{o\}\right\rangle$. Then there is an $(s+1) \leq$-rnc $\operatorname{arc} B$ such that $B \supset A$, each connected components of $B$ has degree $s+1$ and $B_{\text {red }}=A_{\text {red }}$.

Proof. Since $o \notin A_{\text {red }}$ and $A \cup\{o\}$ is in linear general position, for each $a \in A_{\text {red }}$ the linear spaces $W_{a}:=\left\langle A_{a}\right\rangle$ and $V_{a}$ are defined over $K, \operatorname{dim} V_{a}=s$, and $W_{a}$ is a hyperplane of $W_{a}$. By assumption $A_{a}$ in a connected degree $s$ rnc arc of $W_{a}$.

By Remark $2.7 A_{a}$ may be extended to a connected degree $s+1 \mathrm{rnc}$ arc over $\mathbb{F}_{q}$ spanning $V_{a}$. Set $B:=\cup_{a \in A_{\text {red }}} B a$. To conclude the proof it is sufficient to prove that $B$ is in a linear general position. Fix a proper linear subspace $V$ defined over $K$ and assume $\operatorname{deg}(B \cap V) \geq \operatorname{dim} V+2$. Since $A$ is in linear general position, $V \cap A \varsubsetneqq V \cap B$. Since each connected rnc scheme of degree $s+1$ contains a unique subscheme of degree $s, \operatorname{deg}(B \cap V)-\operatorname{deg}(B \cap A)$ is the number, $x$, of the points $a_{1}, \ldots, a_{x} \in A_{\text {red }}$ such that $B_{a_{i}} \subseteq B \cap A$ and that $B_{a_{i}} \subset V$ if and only if $V_{a} \subseteq V$. 
First assume $x=1$. Since $A \cup\{o\}$ is an $\operatorname{arc}, \operatorname{deg}(B \cap V)=\operatorname{deg}((A \cup\{o\}) \cap V) \leq \operatorname{dim} V+1$, a contradiction.

Now assume $x \geq 2$. In this case $V \supseteq W_{a_{1}} \cup W_{a_{2}} \cup\{o\}$. Since $s \geq[r / 2]$ and $A_{a_{1}} \cup A_{a_{2}} \cup\{o\}$ is linearly independent, $V=\mathbb{P}^{r}(\bar{F})$, a contradiction.

Remark 2.7 Let $Z \subset \mathbb{P}^{r}(\bar{F})$ be a zero-dimensional scheme such that all its connected components are defined over $K$. Let $W \subseteq Z$ be a connected component of $Z$. Let $o \in \mathbb{P}^{r}(K)$ be the reduction of $W$. Assume $w:=\operatorname{deg}(W) \leq r$. There is a rational normal curve $C \subset \mathbb{P}^{r}(\bar{F})$ defined over K such that $o \in C(K)$ and $W$ is the effective divisor wo of $C(\bar{F})$ if and only if there is a $(w-1)$-dimensional linear subspace $V$ of $\mathbb{P}^{r}(\bar{F})$ containing $o$ and a rational normal curve $C^{\prime}$ of $V$ defined over $K$ such that $o \in C^{\prime}(K)$ and $W$ is the effective divisor wo of $C^{\prime}(\bar{F})$.

Lemma 2.8 Let $Z \subset \mathbb{P}^{r}(\bar{F}), r \geq 2$, be a zero-dimensional scheme whose connected components are curvilinear and defined over $K$. The following conditions are equivalent:

(1) $Z$ is an arc with respect to $K$;

(2) $\operatorname{deg}(V \cap Z) \leq \operatorname{dim} V+1$ for each proper subspace $V \subset \mathbb{P}^{r}(\bar{F})$ defined over $\bar{F}$;

(3) there is a field $K^{\prime} \supseteq K$ such that $\operatorname{deg}(Z \cap V) \leq \operatorname{dim} V+1$ for each proper linear subspace of $\mathbb{P}^{r}(\bar{F})$ defined over $K^{\prime}$;

(4) $\operatorname{deg}(Z \cap V) \leq \operatorname{dim} V$ for each proper linear subspace $V \subset \mathbb{P}^{r}(\bar{F})$ defined over a field containing $K$.

Proof. Obviously (4) $\Rightarrow(2) \Rightarrow(1)$. Condition (3) does not require that $K^{\prime}$ is algebraic over $K$, but (2) implies a similar statement for any algebraically closed field containing $\mathrm{K}$ instead of $\bar{F}$. Thus it is sufficient to prove (4) $\Rightarrow(1)$. Fix a field $K^{\prime} \supseteq K$ and assume $\operatorname{deg}(Z \cap V) \geq \operatorname{dim} V+2$ for some proper linear subspace defined over $K^{\prime}$. Set $s:=\#\left(Z_{\text {red }} \cap\right.$ $L)$ and write $Z_{\text {red }} \cap L \cap\left\{o_{1}, \ldots, o_{s}\right\}$ with $Z_{o_{i}}$ the connected component of $Z$ with $o_{i}$ as its reduction. Since $Z_{o_{i}}$ and $V$ are defined over $K^{\prime}, Z_{i}^{\prime}:=Z_{o_{i}} \cap K^{\prime}$ is defined over $K^{\prime}$. However, since each subscheme of $Z_{o_{i}}$ is defined over $K, Z_{i}^{\prime}$ is defined over $K$. Thus the linear space $W:=\left\langle Z_{i}^{\prime} \cup \cdots \cup Z_{s}^{\prime}\right\rangle$ is defined over $K$. Since $Z \cap V=Z \cap W, Z$ is not an arc.

Definition 2.9 Assume $K$ perfect. Let $V \subset \mathbb{P}^{n}(\bar{F})$ be an $x$-dimensional vector space. An $x$-dimensional osculating data defined over $K$ spanning $V$ is a degree $x+1$ connected curvilinear scheme $Z$ such that $V$ is the minimal linear space containing $Z$.

Remark 2.10 We recall that if a zero-dimensional scheme $Z$ defined over $K$ is curvilinear and connected, then for each integer $z \in\{1, \ldots, \operatorname{deg}(Z)\}$ there is a unique degree $z$ zero-dimensional scheme $W \subset Z$. If $K$ is perfect the uniqueness of $W$ implies that $W$ is defined over $K$ by Galois theory. Thus if $K$ is perfect Definitions 1.4 and 2.9 are equivalent.

Lemma 2.11 Assume $K$ perfect. Let $V \subset \mathbb{P}^{n}(\bar{F})$ be an $x$-dimensional vector space. Fix the field $K$. There is a surjection $j$ between the $x$-osculating data over $K$ of Definition 1.3 and the ones of Definition 1.4 and $j(C, o)=j\left(C^{\prime}, o\right.$ ') implies $o^{\prime}=o$.

Proof. Take $(C, o)$ as in Definition 1.3. The scheme $Z$ which represents the degree $x+1$ effective divisor $(x+1) o$ of $C$ satisfies all the assumptions of Definition 1.4 with for any $z \in\left\{1, \ldots, x_{1}\right\}$ the effective divisor $z o$ of $C$ the only degree $z$ subscheme of $Z$. Now take $Z$ satisfying Definition 1.4 and let $Z_{1}, \ldots, Z_{x+1}$ the closed subschemes of $Z$ of degree $\{1, \ldots$, $x+1$ \} of $Z$. Thus $Z_{1}$ is a point, $o, Z_{2}$ spans a line $V_{1}$ and so on with $Z_{z}$ spanning the $(z-1)$-dimensional linear space $V_{z_{1}}$. We get a complete flag of $V$ defined over $K$. This flag gives a system of homogeneous coordinates $z_{0}, \ldots, z_{x}$ of $V$ with $o=$ $[1: 0: \cdots: 0], V_{1}$ spanned by $[0: 1: 0, \cdots: 0]$ and $o$ and so on. This system of coordinates determines a rational normal curve $C$ with $Z=(x+1) o$.

Remark 2.12 If $Z$ is an $x$-dimensional osculating data for $V$ in the sense of Definition 1.4, then for each $z \in\{1, \ldots$, $x+1\}$ the only degree $z$ subscheme $Z$ of $Z$ spans a $(z-1)$-dimensional linear subspace of the linear space spanned by $Z$.

Remark 2.13 Let $X \subset \mathbb{P}^{n}(\bar{F})$ be any rational normal curve defined over $K$. Any degree $n+1$ zero-dimensional subscheme of $X(\bar{F})$ is linearly independent, i.e. it spans $\mathbb{P}^{n}(\bar{F})$. Any degree $t \leq n$ zero-dimensional subscheme $Z \subset X(\bar{F})$ spans a $(t-1)$-dimensional linear subspace defined over any field $K_{1}$ such that $Z$ is defined over $K_{1}$.

\section{3. $\mathbb{P}^{2}\left(\mathbb{F}_{q}\right)$}

In this section, we take $F=\mathbb{F}_{p}, K=\mathbb{F}_{q}$ and $r=2$.

Proposition 3.1 Let $S \subset \mathbb{P}^{2}\left(\mathbb{F}_{q}\right)$ be an arc. If q is even assume $\# S \leq q+1$. For each $o \in S$ fix any tangent line $L_{o} \subset$ 
$\mathbb{P}^{2}\left(\mathbb{F}_{q}\right)$ to $S$ and set $v_{o}:=\left(2 o, L_{o}\right)$ and $Z:=\cup_{o \in S^{v_{o}}}$. Then $Z$ is an arc.

Proof. By a theorem of Segre B $\# S \leq q+1$ if $q$ is odd ([9], [12, p. 869-861]) and hence for all $q$ each $o \in S$ is contained in $q+2-\# S>0$ unisecant lines of $S$. Thus $Z$ is well-defined. Take a degree 3 subscheme $W \subseteq Z$ and assume that $W$ is contained in a line. Since $S$ is an arc, $\#(L \cap S) \leq 2$. Since each connected component of $Z$ has degree $\leq 2$, \# $(S \cap L)$ $=2$ and $W=\{o\} \cup v_{o^{\prime}}$ for some $o, o^{\prime} \in S$ such that $o \neq o^{\prime}$. Since $\left\{o, o^{\prime}\right\} \subseteq \mathbb{P}^{2}\left(\mathbb{F}_{q}\right), L$ is defined over $\mathbb{F}_{q}$. Since $\#(S \cap L) \geq 2$, $L$ is not a unisecant line of $S$. Thus $v_{o^{\prime}} \nsubseteq L(\bar{F})$, a contradiction.

Proof of Theorem 1.8 Proposition 3.1 shows that $z=2 q+2$ if $q$ is odd (by the classification of ovals for $q$ odd proved by Segre B ([9], [12, p. 869-861]) and $2 q+2 \leq z \leq 2 q+4$ if $q$ is even. Assume $q$ even. To exclude the case $z \in\{2 q$ $+3,2 q+4\}$ and that the maximal degree arc are obtained by the recipe of Proposition 3.1 it is sufficient to use [6, Cor. 13.41] or the case $n=2$ of [5, Th. 12.2.3]. By [6, Cor. 13.41] or the case $n=2$ of [5, Th. 12.2.3] a degree $q+2 \operatorname{arc}$ is not strictly contained in a + rnc arc over $\mathbb{F}_{q}$.

Proposition 3.2 Take $A \subset \mathbb{P}^{2}\left(\mathbb{F}_{q}\right)$ which is an arc. Set $w:=\# A$. If $p=2$ assume $\# A \leq q+1$, so that each point of $A$ is contained in $q+2-\# A$ unisecant lines of $A$. For each $o \in A$ fix a unisecant line $L_{o}$ of A containing $o$. Let $v_{o}$ be the degree 2 zero-dimensional subscheme of $L_{o}\left(\mathbb{F}_{p}\right)$ with $o$ as its reduction. Set $Z:=\cup_{o \in A^{v} v}$. Then $\operatorname{deg}(Z)=2 w, Z$ is rnc and $Z$ is an arc. Moreover, if $A$ is a complete arc, there is no $\operatorname{arc} Z^{\prime} \supsetneq Z$ whose connected components have degree $\leq 2$.

Proof. Since $L_{o}$ is unisecant to $A, L_{o} \cap A=\{o\}$ for all $o$ and all $L_{o}$. Thus $\operatorname{deg}(Z)=2 w$. Remark 2.7 shows that $Z$ is rnc. Assume the existence $Z^{\prime} \supsetneq Z$ whose connected components have degree $\leq 2$. Thus the reduction of $Z^{\prime}$ contains $a \in$ $\mathbb{P}^{2}\left(\mathbb{F}_{q}\right) \backslash A$. Since any subscheme of an arc is an arc, $A \cup\{a\}$ is an arc. Thus $A$ is not completed.

\section{4. $\mathbb{P}^{r}\left(\mathbb{F}_{q}\right), r>2$}

In this section, we take $F=\mathbb{F}_{p}, K=\mathbb{F}_{q}, \bar{F}=\bar{F}_{p}$ and $r \geq 3$. Set $m(2, q):=q+2$ if $q$ is even and $m(2, q):=q+1$ if $q$ is odd, i.e. let $m(2, q)$ be the maximal cardinality of an $\operatorname{arc}$ of $\mathbb{P}^{2}\left(\mathbb{F}_{q}\right)\left(\left[6\right.\right.$, Th. 13.32 and 13.37], [7, p. 284]). Let $m^{\prime}(2, q)$ denote the second highest cardinality of a complete $\operatorname{arc}$ of $\mathbb{P}^{2}\left(\mathbb{F}_{q}\right)([5$, p. 286], [6, Th. 13.48 and §13.7]).

Remark 4.1 Assume $q>r>2$. The MDS conjecture asks if the maximal cardinality $m(r, q)$ of an arc of $\mathbb{P}^{r}\left(\mathbb{F}_{q}\right)$ is $q+1$. Moreover, it is expected that all arcs with cardinality $q+1$ are (the $\mathbb{F}_{q}$-points of) rational normal curves. We have $m(4, q)=q+1$ ([7, Th. 27.6.3]), $m(r, q)=q+1$ if $r>p$ and in particular if $q=p$ ([2], [3, Th. 32]) or if $q \geq 2 r-2$ and $q \neq p$, then $a=q+1$ ([3, Th. 35]). Moreover, if $r<p$ and $r \neq(q+1) / 2$ every maximal arc is the set of $\mathbb{F}_{q}$-points of a rational normal curve ([3, Th. 36]). Moreover, if $r=3$ and $q$ is odd, every maximal arc is a rational normal curve.

Lemma 4.2 Let $A \subset \mathbb{P}^{r}\left(\mathbb{F}_{q}\right)$ be a finite set which is an arc. Assume $\# A>m^{\prime}(2, q)$ and the existence of $o \in A$ and a degree $(r-1)$ connected rnc scheme $E \subset \mathbb{P}^{r}(\bar{F})$ defined over $\mathbb{F}_{q}$ and with $E \cup A$ an arc and that $E \cup A$ is a weakly complete arc. Then $\# A=m(2, q)$.

Proof. Let $V$ be the $(r-2)$-dimensional linear space spanned by $E$ and $V^{\prime} \subset V$ the linear subspace spanned by the degree $(r-2)$ subscheme $E^{\prime}$ of $E$. Let $l_{V^{\prime}}: \mathbb{P}^{r}(\bar{F}) \backslash V^{\prime} \rightarrow \mathbb{P}^{2}(\bar{F})$ denote the linear projection from $V^{\prime} . V$ and $V^{\prime}$ are defined over $\mathbb{F}_{q}$ and hence $l_{V^{\prime}}$ induces a surjection $l: \mathbb{P}^{r}\left(\mathbb{F}_{q}\right) \backslash V^{\prime}\left(\mathbb{F}_{q}\right) \rightarrow \mathbb{P}^{2}\left(\mathbb{F}_{q}\right)$. Since $E^{\prime} \cup(A \backslash\{o\})$ is an arc, $V^{\prime} \cap A=\{o\}$. Thus $A^{\prime}$ $:=l(A \backslash\{o\})$ is an arc with cardinality $\# A-1$. Since $V$ and $V^{\prime}$ are defined over $\mathbb{F}_{q}, l_{V^{\prime}}\left(V \backslash V^{\prime}\right)$ is a point $a \in \mathbb{P}^{2}\left(\mathbb{F}_{q}\right)$. Since $E$ $\cup A$ is an arc, $A^{\prime \prime}:=A^{\prime} \cup\{a\}$ is an arc and $\#\left(A^{\prime} \cup\{a\}\right)=\# A$.

Claim $1 A^{\prime \prime}$ is a complete arc.

Proof of Claim 1 Assume that $A^{\prime \prime}$ is not a complete arc and take $a \in \mathbb{P}^{2}\left(\mathbb{F}_{q}\right) \backslash A^{\prime \prime}$ such that $A^{\prime \prime} \cup\{a\}$ is an arc. Take any $b \in \mathbb{P}^{r}\left(\mathbb{F}_{q}\right) \backslash V^{\prime}\left(\mathbb{F}_{q}\right)$ such that $l(b)=a$. Since $A^{\prime \prime} \cup\{a\}$ and $E \cup\{a\}$ are arcs, $E \cup A \cup\{b\}$ is an arc, contradicting the weak completeness of $A \cup E$.

Since $\# A>m^{\prime}(2, q)$ and $A^{\prime \prime}$ is a complete arc (Claim 1), $\# A=m(2, q)$.

Lemma 4.3 Let $A \subset \mathbb{P}^{r}\left(\mathbb{F}_{q}\right)$ be an arc such that $\# A \geq m^{\prime}(2, q)+2$. Assume the existence of $o \in A$ and a degree $(r-2)$ connected rnc scheme $E \subset \mathbb{P}^{r}(\bar{F})$ defined over $q$ and such that $E \cup A$ is an arc. Then $\# A=m(2, q)$.

Proof. Let $V^{\prime}$ be the linear space spanned by $E$. As in the proof of Lemma 4.2 it is sufficient to observe that the arc $l(A \backslash\{O\})$ has cardinality $>m^{\prime}(2, q)$. 
Lemmas 4.2 and 4.3 may be generalized in the following way.

Lemma 4.4 Fix $(s, e) \in \mathbb{N}^{2} \backslash\{(0,0)\}$ and positive integers $r_{1}, \ldots, r_{s}, m_{1}, \ldots, m_{e}$ such that $r_{1}+\ldots+r_{s}+m_{1}+\ldots+$ $m_{e}=r-1+e$. Let $A \subset \mathbb{P}^{r}\left(\mathbb{F}_{q}\right)$ be an arc. Assume the existence of $s+m$ distinct points $o_{1}, \ldots, o_{a}, a_{1}, \ldots, a_{e}$ of $A$, rnc schemes $E_{1}, \ldots, E_{s}, F_{1}, \ldots, F_{e}$ such that $E_{i \text { red }}=\left\{o_{i}\right\}$ for all $i, F_{i \text { red }}=\left\{a_{i}\right\}$ for all $i, \operatorname{deg}\left(E_{i}\right)=r_{i}+1$ for all $i, \operatorname{deg}\left(F_{i}\right)=m_{i}+$ 1 for all $i$ and $A \cup E_{1} \cup \cdots \cup E_{s} \cup F_{1} \cup \cdots \cup F_{e}$ is a weakly complete arc. If $\# A>m^{\prime}(2, q)+e$, then $\# A=m(2, q)+e$.

Proof. Let $V_{i}$ be the linear span of $E_{i}, V_{i}^{\prime}$ the linear span of the unique $\operatorname{deg}\left(E_{i}\right)-1$ subscheme of $E_{i}$, and $W_{i}$ the linear span of $F_{i}$. Let $W$ be the linear space spanned by $V_{1}^{\prime} \cup \cdots \cup V_{s}^{\prime} \cup W_{1} \cup \cdots \cup W_{e}$. The linear space $W$ is defined over $\mathbb{F}_{q}$ and $\operatorname{dim} W=r-3$. Let $l_{w}: \mathbb{P}^{r}(\bar{F}) \backslash W \rightarrow \mathbb{P}^{2}(\bar{F})$ denote the linear projection from $W$. Since $W$ is defined over $\mathbb{F}_{q}$, $l_{w}$ is defined over $\mathbb{F}_{q}$. Thus, if $Z \subset \mathbb{P}^{r}(\bar{F}) \backslash W$ is a zero-dimensional scheme defined over $\mathbb{F}_{q}$, then $l_{w}(Z)$ is defined over $\mathbb{F}_{q}$. Call o $o_{i}^{\prime}, 1 \leq i \leq e$, the image of $l_{w}\left(E_{i} \backslash F_{i}\right)$. As in the proof of Lemmas 4.2 and 4.3 we see that $l\left(A \backslash\left\{o_{1}, \ldots, o_{e}\right\}\right) \cup\left\{o_{1}^{\prime}, \ldots, o_{e}^{\prime}\right\}$ is an arc with cardinality $\# A>m^{\prime}(2, q)$. Thus, $\# A=m(2, q)$.

Proof of Theorem 1.9 Since the case $r=2$ is true by Theorem 1.8, we may assume $r>2$. Let $C$ be a rational normal curve defined over $\mathbb{F}_{q}$. Let $G$ be the degree $r(q+1)$ rnc schemes with $G_{\text {red }}=C\left(\mathbb{F}_{q}\right)$ and such that for each $o \in$ $C\left(\mathbb{F}_{q}\right)$ the connected component of $G$ with $o$ as its reduction is the degree $r$ subscheme of $C$ with $\{o\}$ as its reduction. Since any degree $\leq r+1$ zero-dimensional subscheme of $C(\bar{F})$ is linearly independent, $G$ is an arc. Thus, $z \geq r(q+1)$. Take an $\operatorname{rnc} \operatorname{arc} Z$ with $\operatorname{deg}(Z)=z$ and set $A:=Z_{\text {red }}$ and $a:=\# A$. The maximality of $z$ implies that $Z$ is a complete arc. Hence $Z$ is a weakly complete arc. Since $z>r+1$, each connected components of $Z$ has degree $\leq r$ (Remark 2.12). Thus $z \leq r a$. Hence $a \geq q+1$. The trivial upper bound for arcs in finite projective spaces gives $a \leq q+r$ ([3, Theorem 13]). Since $r \geq 3$ and $q \geq r, r(q+1) \geq q+2 r$. Thus, we may apply Lemma 4.4 if we check the weak completeness of the arc.

The weak completeness of $Z$ follows from the maximality of the integer $z$.

\section{Caps}

Let $Z \subset \mathbb{P}^{r}(\bar{F}), r \geq 2$, be a zero-dimensional scheme. We say that $Z$ is a cap over $K$ if each connected component of $Z$ is defined over $K$ and $\operatorname{deg}(Z \cap L) \leq 2$ for each line $L$ defined over $K$. For any connected rnc scheme $A$ defined over $\mathbb{F}_{q}$ and any positive integer $t$ set $A[t]:=A$ if $\operatorname{deg}(A) \leq t$, while let $A[t]$ be the only degree $t$ subscheme of $A$ with degree $t$ if $\operatorname{deg}(A)>t$. $A[t]$ is defined over $\mathbb{F}_{q}($ Remark 2.2).

Lemma 5.1 Let $Z \subset \mathbb{P}^{r}(\bar{F}), r \geq 2$, be a zero-dimensional scheme whose connected components are curvilinear and defined over $K$. The following conditions are equivalent:

(1) $Z$ is a cap;

(2) $\operatorname{deg}(L \cap Z) \leq 2$ for all lines $L \subset \mathbb{P}^{r}(\bar{F})$ defined over $\bar{F}$;

(3) there is a field $K^{\prime} \supseteq K$ such that $\operatorname{deg}(Z \cap L) \leq 2$ for each line $L$ defined over $K^{\prime}$;

(4) $\operatorname{deg}(Z \cap V) \leq 2$ for each line $L$ defined over a field containing $K$.

Proof. Obviously (2) $\Rightarrow(3),(4) \Rightarrow(2),(4) \Rightarrow(3)$ and (4) $\Rightarrow(1)$. Thus it is sufficient to prove that (1) $\Rightarrow(4)$. Fix a field $K^{\prime} \supseteq K$ and assume $\operatorname{deg}(Z \cap L) \geq 3$ for some line defined over $K^{\prime}$. Set $s:=\#\left(Z_{\text {red }} \cap L\right)$ and write $Z_{\text {red }} \cap L=\left\{o_{1}, \ldots\right.$, $\left.o_{s}\right\}$ with $Z_{o_{i}}$ the connected component of $Z$ with $o_{i}$ as its reduction. Since each connected component of $Z$ is defined over $K, o_{i} \in \mathbb{P}^{r}(K)$ for all $i$. If $s \geq 2$, then $L$ is spanned by $o_{1}$ and $o_{2}$ and hence it is defined over $K$. Now assume $s=1$. In this case, we use that the degree 3 subscheme of $Z_{o_{1}}$ is defined over $K$.

Lemma 5.2 Let $Z \subset \mathbb{P}^{r}(\bar{F})$, be a zero-dimensional scheme whose connected components are defined over $K$, they are rnc schemes and no one of them of degree $>2$ is contained in a line. $Z$ is a cap if and only if its 2-restriction $Z[2]$ is a cap.

Proof. If $Z$ is a cap, then $Z[2]$ is a cap, because it is contained in $Z$ and each connected component of $Z[2]$ is defined over $K$. Fix a line $L \subset \mathbb{P}^{r}(\bar{F})$ and assume $\operatorname{deg}(Z \cap L) \geq 3$. Set $\left\{o_{1}, \ldots, o_{s}\right\}:=Z_{\text {red }} \cap L$. Call $Z_{o_{i}}$ the connected component of $Z$ containing oi. Set $a_{i}:=\operatorname{deg}(Z \cap L)$. Note that $\operatorname{deg}\left(Z_{o_{i}}[2] \cap L\right)=\min \left\{2, a_{i}\right\}$. Each $Z_{o_{i}}$ is rnc and it is contained in a line if and only if $\operatorname{deg}\left(Z_{o_{i}}\right) \leq 2$. If $\operatorname{deg}\left(Z_{o_{i}}\right) \geq 3$, then $\operatorname{deg}\left(Z_{o_{i}} \cap L\right)=\operatorname{deg}\left(Z_{o_{i}}[2] \cap L\right.$. Thus, $\operatorname{deg}(Z \cap L)=\operatorname{deg}(Z[2] \cap L)$.

Remark 5.3 In Lemma 5.2, we allowed connected rnc schemes of arbitrary length as connected components, if 
not collinear. This is the reason why we think the general definition is not very interesting unless we impose that all the connected components have degree $\leq 2$.

\section{References}

[1] Akça Z. A numerical computation of k-3 arcs in the left semifield plane of order 9. International Electronic Journal of Geometry. 2011; 4: 13-20.

[2] Ball S. On sets of vectors of a finite vector space in which every subset of basis size is a basis. Journal of the European Mathematical Society. 2012; 14: 733-748.

[3] Ball S, Lawrauw M. Arcs in finite projective spaces. EMS Surveys in Mathematical Sciences. 2019; 1-2: 133-172.

[4] Ball S, Lawrauw M. Arcs and tensors. Designs, Codes and Cryptography. 2020; 88(1): 17-31.

[5] Hirschfeld JWP. Projective Geometries over Finite Fields. 2nd ed. Oxford University Press; 1998.

[6] Hirschfeld JWP, Korchmàros G, Torres F. Algebraic curves over a finite field. Princeton and Oxford: Princeton Series in Applied Mathematics. Princeton University Press; 2008.

[7] Hirschfeld JWP, Thas JA. General Galois geometries. Oxford Mathematical Monographs. The Clarendon Press Oxford University Press, Oxford Science Publications, New York; 1991.

[8] Hirschfeld JWP, Thas JA. Open problems in finite projective spaces. Finite Fields and their Application. 2015; 32: 44-81.

[9] Segre B. Ovals in a finite projective plane. Canadian Journal of Mathematics. 1954; 7: 414-416.

[10] Segre B. Curve razionali normali ek-archi negli spazi finiti. Annali di Matematica Pura ed Applicata. 1955; 39(1): 357-379.

[11] Segre B. Introduction to Galois geometrie. In: Hirschfeld JWP. (ed.) Atti della Accademia Nazionale dei Lincei, Memorie. 1967; 8: 133-216.

[12] Segre B. Opere scelte. Vol. III, Rome: Edizione Cremonese; 2000.

[13] Storme L, Van Maldeghem H. Primitive arcs in PG(2, q). Journal of Combinatorial Theory Series(A). 1995; 69: 200-216.

[14] Arbarello E, Cornalba M, Griffths P, Harris JD. Geometry of Algebraic curves. Berlin: Springer; 1985.

[15] Rathmann J. The uniform position principle for curves in characteristicp. Mathematische Annalen. 1987; 276: 565579.

[16] Ballico E. The stratification by rank for homogeneous polynomials with border rank 5 which essentially depend on at least 5 variables. Acta Mathematica Vietnaminica. 2017; 42: 509-531.

[17] Ballico E, Bernardi A. Decomposition of homogeneous polynomials with low rank. Mathematische Zeitschrift. 2012; 271: 1141-1149.

[18] Ballico E, Bernardi A. A partial stratification of secant varieties of Veronese varieties via curvilinear subschemes. Sarajevo Journal of Mathematics. 2012; 8: 33-52.

[19] Ballico E, Bernardi A. Tensor ranks on tangent developable of Segre varieties. Linear and Multilinear Algebra. 2013; 61: 881-894.

[20] Ballico E, Bernardi A. Stratification of the fourth secant variety of Veronese variety via the symmetric rank. Advances in Pure and Applied Mathematics. 2013; 4: 215-250.

[21] Ballico E, Bernardi A, Christandl M, Gesmundo F. On the partially symmetric rank of tensor products of W-states and other symmetric tensors. Atti della Accademia Nazionale dei Lincei. Rendiconti Lincei. Matematica e Applicazioni. 2019; 30: 93-124.

[22] Ballico E, Bernardi A, Gesmundo F. A note on the cactus rank for Segre-Veronese varieties. Journal of Algebra. 2019; 526: 6-11.

[23] Bernardi A, Brachat J, Mourrain B. A comparison of different notions of ranks of symmetric tensors. Linear Algebra and its Applications. 2014; 460: 205-230.

[24] Bernardi A, Jelisiejew J, Marques PM, Ranestad K. On polynomials with given Hilbert function and applica-tions. Collectanea Mathematica. 2018; 69: 39-64.

[25] Bernardi A, Gimigliano A, Idà M. Computing symmetric rank for symmetric tensors. Journal of Symbolic Computation. 2011; 46: 34-55.

[26] Bernardi A, Ranestad K. On the cactus rank of cubic forms. Journal of Symbolic Computation. 2013; 50: $291-297$.

[27] Buczyńska W, Buczyński J. Secant varieties to high degree Veronese reembeddings, catalecticant matrices and smoothable Gorenstein schemes. Journal of Algebraic Geometry. 2014; 23(1): 63-90. 
[28] Buczyński J, Ginenski A, Landsberg JM. Determinantal equations for secant varieties and the Eisenbud-KohStillman conjecture. Journal of the London Mathematical Society. 2013; 88: 1-24.

[29] Buczyński J, Jelisiejew J. Finite schemes and secant varieties over arbitrary characteristic. Differential Geometry and its Applications. 2017; 55: 13-67.

[30] Buczyński J, Landsberg JM. Ranks of tensors and a generalization of secant varieties. Linear Algebra and its Applications. 2013; 438: 668-689.

[31] Buczyński J, Landsberg JM. On the third secant variety. Journal of Algebraic Combinatorics. 2014; 40: 475-502.

[32] Ranestad K, Schreyer FO. On the rank of a symmetric form. Journal of Algebra. 2011; 346(1): 340-342.

[33] Adlandsvik B. Joins and higher secant varieties. Mathematica Scandinavica. 1987; 61: 213-222. 